\title{
Development of a 1-THz 4th-Harmonic Gyrotron
}

\author{
Fan-Hong Li, Chao-Hai Du, Zi-Chao Gao,
Shi Pan, Juan-Feng Zhu, Zi-Wen Zhang, Si-Qi Li \\ Fan-Hong Li, Chao-Hai Du, Zi-Chao Gao,
Shi Pan, Juan-Feng Zhu, Zi-Wen Zhang, Si-Qi Li \\ the department of electronics, \\ Peking University, \\ Beijing 100871, China \\ duchaohai@pku.edu.cn
}

\begin{abstract}
A design of a 1-THz $4^{\text {th }}$ Harmonic Gyrotron is proposed in this paper. Fourth harmonic interaction is adopted to lower the strength of the external magnet. The magnet can be as low as 10T. Such a low-intensity magnetic field can be easily proposed by a superconduct magnet, which make the generation of continuous waves at $1 \mathrm{THz}$ possible. Seeing that the mode competition is extremely fierce, a large orbit gun is employed as the driven source. An 80-kV, 0.7-A electron beam is injected into the interaction circuit to excite the fourth-harmonic electromagnetic waves. According to the cold dispersive diagram, the $T_{4,8}$ mode of the cylindrical waveguide is selected as the operating mode to further suppress the mode competition induced by the fundamental modes, the $2^{\text {nd }}$ harmonic modes and the $3^{\text {rd }}$ harmonic modes. It could be inferred based on the multi-mode time-domain theory that an output power of $1.15 \mathrm{~kW}$ can be achieved. The scheme diagram for the hot test is proposed. The LOG is chosen as the electron gun. Particular structure is employed for improving the output efficiency in the interaction circuit.
\end{abstract}

\section{INTRODUCTION}

Terahertz $(\mathrm{THz})$ waves play a critical role in a wide range of potential applications, e.g., plasma fusion diagnostics [1] and dynamic nuclear polarization enhanced nuclear magnetic resonance[2]. However, the development of the $\mathrm{THz}$ technologies is limited by the generation of $\mathrm{THz}$ waves. Gyrotrons, based on the electron cyclotron maser, have the capability of outputting high power and high frequency electromagnetic energy. Existing research have proved the feasibility of generating electromagnetic waves beyond $1 \mathrm{THz}$. A second-harmonic, $1.005-\mathrm{THz}$ gyrotron was confirmed experimentally with a $\mathrm{TE}_{6,11}$ mode using a 19.0 - $\mathrm{T}$ pulse magnet at the University of Fukui, Japan [3]. The experiment designed, manufactured and tested at the Institute of Applied Physics, Russia Academy of Sciences, generated $1.5 \mathrm{~kW}$ of coherent radiation at $1.022 \mathrm{THz}$ using a $38.5-\mathrm{T}$ pulse magnet [4]. The experiments mentioned above both necessitate high strength magnetic field. To achieve the desired peak value of the external magnetic field, such magnets normally produce pulse magnetic field, which means that the generated electromagnetic waves are likely to be short-pulse waves rather than continuous waves. To break the limitation induced by the external magnets, we propose a $4^{\text {th }}$-harmonic gyrotron scheme. The desired intensity of the magnetic field can be further lower, which make it possible that gyrotrons produce $1-\mathrm{THz}$ electromagnetic waves continuously. The paper is organized as follows. In section II, the simulation results are presented. In

\author{
Liang Zhang, \\ Adrian W. Cross \\ the department of physics, SUPA, \\ University of Strathclyde, \\ Glasgow G4 0NG, United Kingdom. \\ a.w.cross@strath.ac.uk
}

section III, the progresses of the experiment are demonstrated. The conclusions are drawn in Section IV.

\section{Simulation Results}

The operating mode is $\mathrm{TE}_{4,8}$ modes. An $80-\mathrm{kV}, 0.7-\mathrm{A}$ electron beam is applied to excite the $4^{\text {th }}$-harmonic gyrotron. The velocity spread is $10 \%$. The pitch factor of the electron gun is 1.5 . The results calculated according to the frequencydomain single-mode theory [5] are shown in Fig. 1. The electron is $7 \%$. $3.49 \%$ of the energy dissipates on the waveguide wall. Thus, the output efficiency is $3.51 \%$.

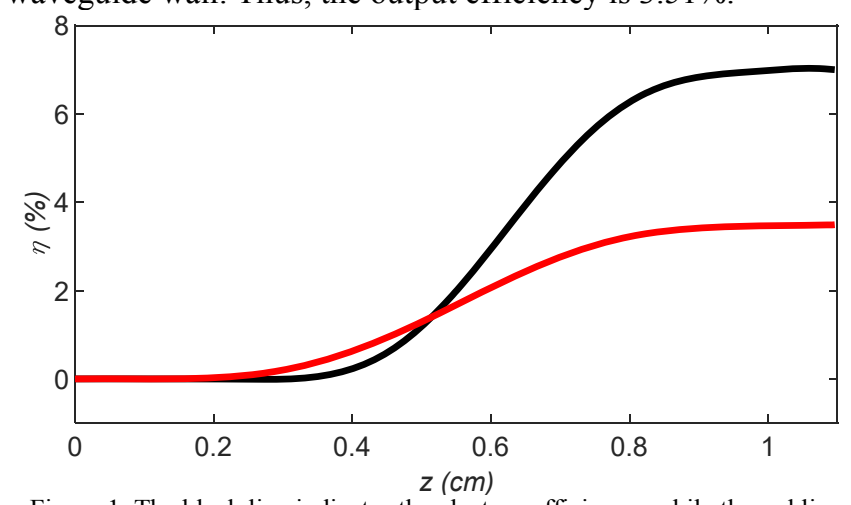

Figure 1. The black line indicates the electron efficiency, while the red line indicates the ohmic loss.

\section{EXPERIMENT PROGRESS}

The experiment design scheme is shown in Fig. 2. The gyrotron designed for experiment is composed of five parts, i.e. the electron gun, the anode, the interaction circuit with liquid cooling, the water-cooled collector and the output window. The LOG is quite different from the MIG. An auxiliary coil is used to produce a zero magnetic field point. The anode of the gyrotron is designed corresponding to the distribution of the external magnetic field. The inner radius of the anode is far more less than the length. Therefore, the anode was split into two parts to improve the co-axiality in fabrication. In hot test, the two anode parts are supported by several frames to keep the co-axiality. In the downstream part of the anode, there remains a ceramic probe. The probe is adopted to observe the current which move through the liquid cooling, the water-cooled collector and the output window. The LOG is quite different from the MIG. An auxiliary coil is used to produce a zero magnetic field point. The anode of the gyrotron is designed corresponding to the distribution of the external magnetic field. The inner radius of the anode is far

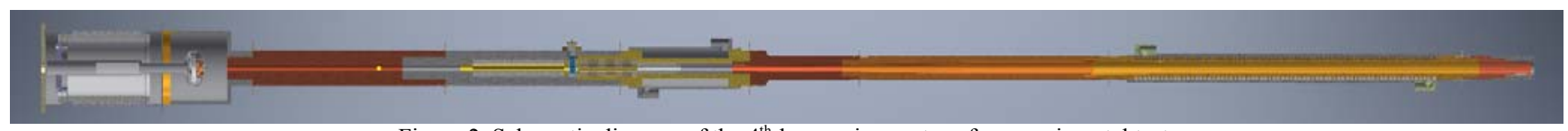

Figure 2. Schematic diagram of the $4^{\text {th }}$-harmonic gyrotron for experimental test. 
external magnetic field. The inner radius of the anode is far more less than the length. Therefore, the anode was split into two parts to improve the co-axiality in fabrication. In hot test, the two anode parts are supported by several frames to keep the co-axiality. In the downstream part of the anode, there remains a ceramic probe. The probe is adopted to observe the current which move through the interaction circuit. In hot test, it's convenient to improve the co-axiality between the cylindrical waveguides and the external magnetic field. The interaction circuit is a two-layer structure. The innermost space is the cylindrical waveguide for beam-wave interaction. The space between two layers is designed as the coolant path. According to our previous study, liquid nitrogen was considered as the coolant. Due to the low efficiency of the $4^{\text {th }}$-harmonic interaction at $1 \mathrm{THz}$, the energy dissipated on the waveguide wall is quantitively low as well. Thus, the liquid nitrogen can be considered to be at boiling temperature all the time. This assumption allows us to avoid many complicated problems in terms of fluid mechanics. The water-cooled collector is design corresponding to the distribution of the external magnetic field. The co-axiality of this part is not likely to be significant. Therefore, each part of the collector is quantitively longer than that of the anode. All spent electrons finally hit the wall of the collector. The energy dissipated is quantitively large than that in the interaction circuit. Thus, fins are employed for better cooling performance.

\section{CONCLUSION}

The experimental scheme diagram of the $1-\mathrm{THz} 4^{\text {th }}$ harmonic gyrotron is demonstrated. The gyrotron has five part, i.e., the electron gun, the anode, the interaction circuit with liquid cooling, the water-cooled collector and the output window. The LOG is employed as the electron gun to excite the electromagnetic waves. For improving the co- axiality in fabrication and hot test, the anode was split into two parts. A two-layer structure is used in the interaction circuit. The liquid nitrogen will be set into the space between two layers to improve the output efficiency. To lower the temperature induced by electrons hit on the wall in collectors, fins is adopted to improve the cooling performance.

\section{ACKNOWLEDGMENT}

Professor Chao-Hai Du would like to thank the Royal Society of London, UK, Newton Advanced Fellowships (NAF\R1\180121) for supporting this work. This work was also in part supported by the National Science Foundation of China under Contract 61861130367, Contract NSAF U1830201, and Contract 61531002.

\section{REFERENCES}

1] N. C. Luhmann Jr, and W. A. Peebles, "Instrumentation for magnetically confined fusion plasma diagnostics,” Rev. Sci. Instrum., vol. 55, pp. 279331, 1984.

[2] Y. Matsuki, K. Ueda, T. Idehara, R. Ikeda, K. Kosuga, I. Ogawa et al., "Application of Continuously Frequency-Tunable $0.4 \mathrm{THz}$ Gyrotron to Dynamic uclear Polarization for $600 \mathrm{MHz}$ Solid-State NMR," Journal of Infrared, Millimeter, and Terahertz Waves, vol. 33, no. 7, pp. 745-755, 2012.

[3] T. Idehara, H. Tsuchiya, O. Watanabe, L. Agusu, and S. Mitsudo, "The first experiment of a $\mathrm{THz}$ gyrotron with a pulse magnet," Int J Infrared Milli, vol. 27, no. 3, pp. 319-331, 2006.

[4] M. Y. Glyavin, A. G. Luchinin, and G. Y. Golubiatnikov, "Generation of $1.5-\mathrm{kW}, 1-\mathrm{THz}$ coherent radiation from a gyrotron with a pulsed magnetic field," Phys Rev Lett, vol. 100, no. 1, p. 015101, Jan 112008.

[5] C.-H. Du, and P.-K. Liu, "Nonlinear full-wave-interaction analysis of a gyrotron-traveling-wave-tube amplifier based on a lossy dielectric-lined circuit," Physics of Plasmas, vol. 17, no. 3, p. 033104, 2010 